\title{
Probing Quantum Gravity through Strong Gravitational Lensing
}

\author{
S Marongwe ${ }^{1}$, \\ Department of Physics and Astronomy \\ Botswana International University of Science and Technology, P. Bag 16, Palapye, \\ Botswana \\ E-mail: stuartmarongwe@gmail.com
}

\begin{abstract}
We report the use of Einstein rings to reveal the quantized and dynamical states of space-time in a region of impressed gravitational field as predicted by the Nexus Paradigm of quantum gravity. This in turn reveals the orbital speeds of objects found therein and the radius of curvature of the quantized space-time. Similarities between the Nexus graviton and the singular isothermal sphere (SIS) in the Cold Dark Matter (CDM) paradigm are highlighted. However unlike the singular isothermal sphere, the Nexus graviton does not contain singularities or divergent integrals. This solves the core cusp problem. In this work, data from a sample of fifteen Einstein rings published on the Cfa-Arizona Space Telescope Lens Survey (CASTLES) website is used to probe the quantized properties of space-time.
\end{abstract}

Keywords: (Quantum Gravity; Gravitational Lensing; Dark Matter; Quantum Vacuum; Graviton)

\section{Introduction}

In 1937, Zwicky (Zwicky 1937 ) provided the first reproducible evidence of the presence of unseen matter in the Coma Cluster group of galaxies by applying the classic virial theorem where he also suggested that the gravitational field of galaxy clusters is expected to deflect the light observed from background galaxies. Today, numerous observations (Boller 1989,

1 To whom any correspondence should be addressed. 
Jauzac et al 2015, Leauthaud et al 2015 , Clowe D et al 2012, Israel et.al, 2014 ) have confirmed the presence of Zwicky's Dark Matter (DM) and most studies (Massey et al 2013, Ellis 2010 , Zhandov et al 2012, Kitching et al, 2015) have used gravitational lensing to explore its distribution in cluster groups. Strong lensing allows the determination of important physical parameters such as the total mass of the lensing object without any assumptions on the dynamics. Einstein rings (ER) are particularly important in constraining the mass within the Einstein radius with great accuracy. Currently, it is thought that DM is the source of much of the lensing potential. Here we report the use of ERs to calculate the quantum state of space-time in the ambient of the baryonic mass. A remarkable feature of the Nexus Paradigm of quantum gravity is that under certain critical conditions, the intrinsic curvature of spacetime in the $n$-th quantum state is the source of deflection and not the curvature due to the presence of baryonic mass. This feature is clearly illustrated in the Bullet Cluster (Clowe et al $2006)$.

\subsection{Theoretical Background}

The nature of DM along with that of Dark Energy (DE) is one of the most perplexing unsolved problems in astrophysics and has largely divided the astrophysical community into some suggesting a modification of the law of gravity (Milgrom 1983, Olmo 2005, Li, Sotiriou \& Barrow 2011, Capozziello 2002) and others suggesting the presence of unseen non-baryonic matter (Goodman \& Witten 1985, Cushman et al 2013, Archambault et al 2009, The CDMS II Collaboration 2010 ). The Nexus Paradigm of quantum gravity (Marongwe 2015, 2017) is a part of a third approach that seeks to explain DM and DE as manifestations of quantum gravity motivated by the fact that both DM and DE manifest themselves thus far, only through the gravitational interaction. Thus DE and DM are 
considered as low energy quantum corrections to GR since these phenomena become apparent at large scales. The current cosmological paradigm, the Lambda Cold Dark Matter (LCDM), is premised on the existence of DE based on Einstein's cosmological constant and DM which is presumed to exist in particulate non-baryonic form whose nature is currently the subject of speculative theories. Thus DE and DM in the LCDM paradigm allow Einstein's field equations to effectively describe galactic and cosmic dynamics.

At galactic scales, the LCDM paradigm faces difficulties in providing a cogent description of galactic rotational curves, (Bullock \& Boylan-Kolchin 2017, McGaugh 2014, Ferrero et al 2012, Van den Bosch \& Swaters 2001) the core cusp problem (De Blok 2009, Navarro, Carlos \& Eke 1996, Se-Heon O et al 2015) and the empirically observed baryonic TullyFisher relation (McGaugh 2011, 2005, Federico, McGaugh \& Schombert 2015, ). At cluster scales LCDM is confronted with challenges in describing the dynamics of the Bullet Cluster (Thompson, Dave \& Nagamine 2014, Lee \& Komatsu 2010) as well as the Abell 520 (Train Wreck) Cluster (Jee et al 2012,2014). These challenges, as well as the null detection of DM (Tan et al 2016 ,Akerib et al 2017, Cushman et al 2013, Sangalard et al. 2005) by both direct and indirect means have motivated other researchers to seek alternatives to the LCDM paradigm. The alternatives should not only address these challenges but explain the success of GR at solar system scales as well.

The Nexus Paradigm of quantum gravity is one of these alternatives and considers space-time as a Nexus Graviton field which can assume $10^{60}$ eigenstates. A Nexus graviton in the $n$-th quantum state as described in Marongwe $(2015,2017)$ is a spherically symmetric pulse or wave packet of four space with the following components

$$
\begin{aligned}
\Delta x_{n}^{\mu} & =\frac{2 r_{H S}}{n \pi} \gamma^{\mu} \int_{-\infty}^{\infty} \operatorname{sinc}\left(k^{\mu} x_{\mu}\right) e^{i k x} d k^{\mu} \\
& =\gamma^{\mu} \int_{-\infty}^{\infty} a_{n k} \varphi_{(n k \mu)} d k^{\mu}
\end{aligned}
$$


Where $\quad \frac{2 r_{H S}}{n \pi}=\sum_{k=-\infty}^{k=+\infty} a_{n k}$

Here $\gamma^{\mu}$ are the Dirac matrices, $r_{H S}$ is the Hubble radius, $\varphi_{(n k \mu)}=\operatorname{sinc}\left(k^{\mu} x_{\mu}\right) e^{i k x}$ are Bloch energy eigenstate functions and $k x=k^{\mu} x_{\mu}$. The Bloch functions can only allow the four wave vector to assume the following quantized values

$$
k^{\mu}=\frac{n \pi}{r_{H S}^{\mu}} n= \pm 1, \pm 2 \ldots 10^{60}
$$

The minimum four radius of the Nexus graviton is the Planck length and the Hubble four radius is the maximum. The $10^{60}$ states arise from the ratio of Hubble four radius to the Planck four length. The Nexus gravitons in each eigenstate of space-time generate an infinite Bravias four lattice.

Each graviton is associated with a conjugate four momentum which can also be expressed as a Fourier integral as

$$
\begin{aligned}
\Delta p_{n}^{\mu} & =\frac{2 n p_{1}}{\pi} \gamma^{\mu} \int_{-\infty}^{\infty} \varphi_{n, k} d k^{\mu} \\
& =\gamma^{\mu} \int_{-\infty}^{\infty} c_{n, k} \varphi_{n, k} d k^{\mu}
\end{aligned}
$$

Where $p_{1}$ is the four momentum of the ground state graviton

Each component of the four displacement vector transforms according to the law

$$
\Delta x_{n}^{\prime \mu}=\exp \left(\frac{1}{8} \omega_{\mu v}\left[\gamma_{\mu}, \gamma_{v}\right]\right) \Delta x_{n}^{\mu}
$$

Where $\omega_{\mu v}$ is an antisymmetric $4 \mathrm{x} 4$ matrix parameterizing the transformation. 
Therefore, each component of the four vector has a spin half. A summation of all the four half spins yields a total spin of 2 for the Nexus Graviton.

From Eqn.(3), the four momentum of the $n$-th state graviton is

$$
(\hbar)^{2} k^{\mu} k_{\mu}=\frac{E_{n}^{2}}{c^{2}}-\frac{3\left(n h H_{0}\right)^{2}}{c^{2}}=0
$$

This can be expressed in terms of the cosmological constant, $\Lambda$ as

$$
\Lambda_{n}=\frac{E_{n}^{2}}{(h c)^{2}}=\frac{3 k_{n}^{2}}{(2 \pi)^{2}}=n^{2} \Lambda
$$

From Eqn.(7), the Nexus graviton can be considered as a compact Reimannian manifold of positive Ricci curvature expressed in the form

$$
G_{(n) \mu v}=n^{2} \Lambda g_{\mu v}
$$

where $G_{(n) \mu \nu}$ is the Einstein tensor of space-time in the $n$-th state. Eqn.(8) depicts a contracting geodesic ball and as explained in Marongwe $(2015,2017)$ this is DM which is an intrinsic curvature of space-time in the $n$-th quantum state. The DE arises from the emission of a ground state ( $n= \pm 1$ ) graviton such that Eqn.(8) becomes

$$
G_{(n) \mu v}=\left(n^{2}-1\right) \Lambda g_{\mu v}
$$

Eqn.(9) can be visualised as a superposition two closed manifolds; one with a positive Ricci curvature $n^{2} \Lambda g_{\mu v}$ causing a contraction and the other with a negative curvature $(-1) \Lambda g_{\mu v}$ causing an expansion of the geodesic ball.

If the graviton field is perturbed by the presence of baryonic matter then Eqn.(9) becomes

$$
G_{(n) \mu v}=k T_{\mu \nu}+\left(n^{2}-1\right) \Lambda g_{\mu v}
$$


The term $\left(n^{2}-1\right) \Lambda g_{\mu v}$ is the quantum correction to GR which replaces the need for DM and DE. This quantum correction gives space-time a superimposed state of having both a positive and a negative Ricci flow.

It is important to note that unlike the standard model graviton the Nexus graviton does not execute translational motion, rather it can only expand by the emission of the ground state Nexus graviton. The ground state graviton is unique among others because it carries a negative metric signature.

The Ricci flow in the form of the Einstein tensor induces motion on any test particle of baryonic matter embedded within the Nexus graviton in a manner dictated by the Strong Equivalence Principle.

From Marongwe (2015) the solution to Eqn. (8) is computed as

$$
d s^{2}=-\left(1-\left(\frac{2}{n^{2}}\right)\right) c^{2} d t^{2}+\left(1-\left(\frac{2}{n^{2}}\right)\right)^{-1} d r^{2}+r^{2}\left(d \theta^{2}+\sin ^{2} \theta d \varphi^{2}\right)
$$

There are no singularities in Eqn.(11). At high energies, characterized by microcosmic scale wavelengths of the Nexus graviton and high values of $n$, space-time is flat although its constituent Nexus gravitons are highly compact. The space-time begins to curve substantially at low energies where the associated constituent Nexus graviton wavelengths are at macrocosmic scales. In the $(n= \pm 1)$ state we notice that the metric signature of Eqn.(11) becomes negative and that space-time is flat. Thus space-time in this quantum state can only expand.

For weak gravitational fields that characterize distances ranging from solar system to cosmic scales, the solution to Eqn.(10) for an aggregation of baryonic matter $M(r)$ within a radius $\mathrm{r}$, as provided in Marongwe (2015) is expressed as 


$$
\frac{d^{2} r}{d t^{2}}=\frac{G M(r)}{r^{2}}+H_{0} v_{n}-H_{0} c
$$

Here $c$ is the speed of light.

The first term on the right is the Newtonian gravitational acceleration, the second term is a radial acceleration induced by space-time in the $n$-th quantum state and the final term is acceleration due to DE. The dynamics becomes strongly non-Newtonian when

$$
\frac{G M(r)}{r^{2}}=H_{0} c=\frac{v_{n}^{2}}{r}
$$

These are conditions in which the space-time curvature due to baryonic matter is annulled by that due to the presence of DE. Under such conditions

$$
r=\frac{v_{n}^{2}}{H_{0} c}
$$

Substituting for $r$ in Eqn. (13) yields

$$
v_{n}^{4}=G M(r) H_{0} c
$$

This is the Baryonic Tully - Fisher relation. The conditions permitting the DE to cancel out the curvature due to baryonic matter leave quantum gravity as the unique source of curvature. Thus condition (13) reduces Eqn.(12) to

$$
\frac{d^{2} r}{d t^{2}}=\frac{d v_{n}}{d t}=H_{0} v_{n}
$$

From which we obtain the following equations of galactic and cosmic evolution

$$
\begin{array}{cl}
r_{n}=\frac{1}{H_{0}} e^{\left(H_{0} t\right)}\left(G M(r) H_{0} c\right)^{\frac{1}{4}} & =\frac{v_{n}}{H_{0}} \\
v_{n}=e^{\left(H_{0} t\right)}\left(G M(r) H_{0} c\right)^{\frac{1}{4}} & =H_{0} r_{n} \\
a_{n}=H_{0} e^{\left(H_{0} t\right)}\left(G M(r) H_{0} c\right)^{\frac{1}{4}} & =H_{0} v_{n}
\end{array}
$$

Here $r_{n}$ is the radius of curvature of space-time in the $n$-th quantum state (which is also the radius of the $n$-th state Nexus graviton), $v_{n}$ the orbital velocity of objects embedded in that space-time, and $a_{n}$, their radial acceleration within it. The amplification of the radius of curvature with time explains the existence of ultra-diffuse galaxies and the spiral shapes of 
most galaxies (see Marongwe 2015, 2017). The increase in orbital velocity with time explains why early type galaxies composed of population II stars are fast rotators. At cosmic scale, Eqn.(18) is a comprehensive form of the Hubble Law. Eqn.(19) explains late time cosmic acceleration which began once condition (13) was satisfied or equivalently from Eqn.(10), when the density of baryonic matter was at the same value as that of DE. Thus condition (13) also explains the Coincidence Problem.

\subsection{Gravitational lensing and quantum gravity}

In the context of the Nexus Paradigm, a gravitational field is a region of space-time in a low quantum state or a zone with a low vacuum expectation value

$$
\left\langle\psi_{n}\left(r_{i}\right)\left|T_{00}\right| \psi_{n}\left(r_{j}\right)\right\rangle=n^{2} \rho_{D E}
$$

where $\psi_{n}$ is the wave function of the quantum vacuum in the $n$-th quantum state and $\rho_{D E}$ is the density of DE. From Marongwe (2015), the flow of space-time in each quantum state of curvature $r_{n}$, induces a constant orbital speed onto any test particle embedded within it of

$$
v_{n}=H_{0} r_{n}=c / n
$$

The deflection of light through gravitational lensing by space-time in the $n$-th quantum state is

$$
\alpha=(\theta-\beta) D_{l s} / D_{s}=4 / n^{2}
$$

Here $D_{1 s}$ is the distance between the lens and the source and $D_{s}$ the distance between the source and the observer.

From Eqn.(22) we obtain

$$
n=2 \sqrt{\frac{D_{s}}{D_{l s}(\theta-\beta)}}
$$

Thus the orbital speed of a particle embedded in the $n$-th quantum state is

$$
v_{n}=c \sqrt{\frac{D_{l s}(\theta-\beta)}{4 D_{s}}}=e^{(H t)}\left(G M(r) H_{0} c\right)^{\frac{1}{4}}
$$

In the case were $\beta=0$ an Einstein ring forms and Eqn.(24) becomes 


$$
v_{n}=c \sqrt{\frac{D_{l s} \theta_{E}}{4 D_{s}}}=e^{(H t)}\left(G M(r) H_{0} c\right)^{\frac{1}{4}}
$$

Here we observe similarities with the cold dark matter (CDM) model where the dispersion velocities for a SIS are calculated from

$$
\sigma=c \sqrt{\frac{D_{l s} \theta_{E}}{4 \pi D_{s}}}
$$

Hence Eqn.(25) is the link between the CDM paradigm and the baryonic Tully-Fisher relation. In such a scenario $r_{n}$, would denote the radius of the CDM halo. However, in the Nexus Paradigm, the CDM is vacuum energy as described by Eqn.(20).Moreover Eqn.(11) describing the Nexus graviton has no singularity nor divergences found in the SIS model.

If the radial velocity and baryonic mass content in the lensing system are known then one can calculate the time $t$, which has elapsed since the lensing system became a system in dynamical equilibrium as stipulated by condition (13).

\subsection{Preliminary test: the simulation of galaxy rotation curves}

In this section we make a preliminary test of the Nexus Paradigm using the observational data on the rotation curve of a galaxy by computing the speed for circular orbits as a function of the distance from the galactic centre. This speed depends on all the mass within a sphere of that radius. Since a spiral galaxy is composed of a central spherical bulge, a disk of gas and stars, a spherical halo of stars. The contributions from each component are summed up.

$$
\mathrm{M}=\mathrm{M}_{\mathrm{stars}}+\mathrm{MHI}_{\mathrm{HI}}+\mathrm{M}_{\mathrm{DB}}
$$


For ease of calculations, a spherically symmetric galaxy is considered such that the density of visible matter, $\rho(r)$, contains an inner core at radius $r=r_{c}$. The acceleration law of galactic kinematics from the Nexus Paradigm is

$$
\frac{v^{2}}{r}=\frac{G M(r)}{r^{2}}+H_{0} v-H_{0} c
$$

From which we find

$$
v=\frac{1}{2} H_{0} r+\frac{\sqrt{H_{0}^{2} r^{2}+4 r\left(\frac{G M(r)}{r^{2}}-H_{0} c\right)}}{2}
$$

This law reduces to $v=e^{\left(H_{0} t\right)}\left(G M(r) H_{0} c\right)^{\frac{1}{4}}=H_{0} r$ once condition $\frac{G M(r)}{r^{2}}=H_{0} c$ in Eqn.(29) has been satisfied.

The total mass $\mathrm{M}(\mathrm{r})$ of a sphere of radius $\mathrm{r}$ is given by the expression

$$
M(r)=4 \pi \int_{0}^{r} r^{2} \rho(r) d r
$$

A simple model of $\mathrm{M}(\mathrm{r})$ is

$$
M(r)=M_{0}\left(\frac{r}{r_{c}+r}\right)^{3 \beta}
$$

Where $\beta=1$ for HSB galaxies and $\beta=2$ for LSB galaxies

Well inside the core radius, the density is constant for HSB galaxies. However, for LSB galaxies the density follows the trend $\rho(r) \propto\left(r / r_{c}\right)^{3}$. For testing the Nexus paradigm we consider the high resolution rotation curves for the LSB galaxies since they provide a clean testing ground for any theory of galaxy rotation curves. For the simulation we apply rotation curve data from Kuzio de Naray et al. (2006, 2008) 


\subsection{The computational method for the rotational curves}

The exponential term in the equation $v=e^{\left(H_{0} t\right)}\left(G M(r) H_{0} c\right)^{\frac{1}{4}}$ is considered to approximate unity for a galactic system that is less than 4 billion years old since it attained dynamic equilibrium. The equation is then reduced to

$$
v=\left(G M(r) H_{0} c\right)^{\frac{1}{4}}
$$

If we know the asymptotic velocity $v_{c}$ from the data then Eqn. (32) for LSB galaxies is reduced to

$$
\begin{aligned}
& v=\left(G M_{0} H_{0} c\right)^{\frac{1}{4}}\left(\left(\frac{r}{r+r_{c}}\right)^{6}\right)^{\frac{1}{4}} \\
& v=v_{c}\left(\left(\frac{r}{r+r_{c}}\right)^{6}\right)^{\frac{1}{4}}
\end{aligned}
$$

Next we input the radius data from Kuzio de Naray et al. $(2006,2008)$ and $r_{c}$ is adjusted to give the best line of fit. Adjusting $r_{c}$ is adjusting the average baryonic density of the galaxy. A large $r_{c}$ implies a low average baryonic mass density while a low $r_{c}$ implies a high average density.

The following are the respective constants in SI units:

Gravitational constant $\mathrm{G}=6.67259 \times 10^{-11} \mathrm{~m}^{3} \mathrm{~kg}^{-1} \mathrm{~s}^{-2}$

1 solar mass $=1.989 \times 10^{30} \mathrm{~kg}, 1$ kilo parsec $=3.086 \times 10^{19} \mathrm{~m}$ Hubble constant $=2.2 \times 10^{-18} \mathrm{~s}^{-1}$ 


\section{Predicted and observed rotational curves}

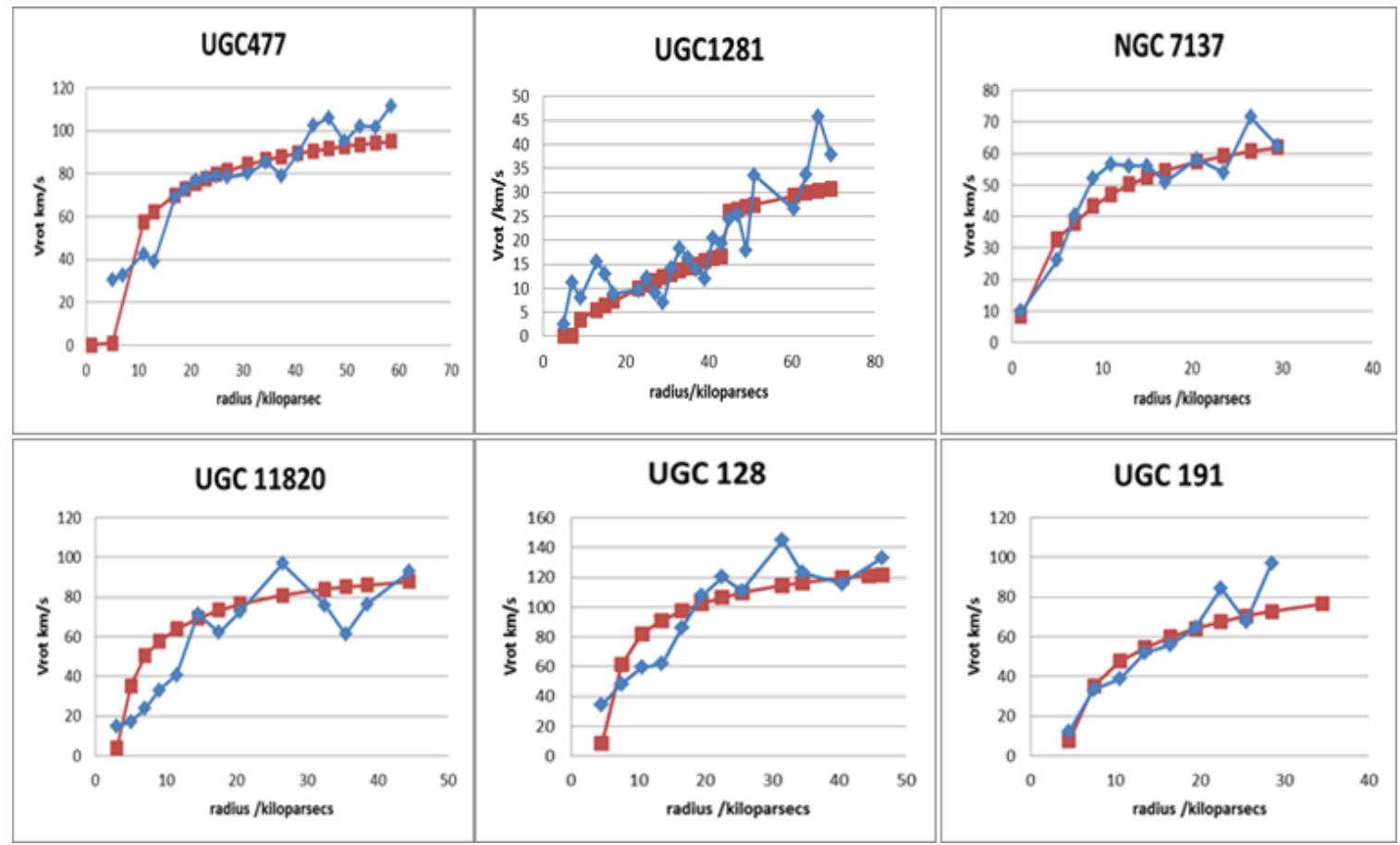

Fig.1

Galaxy rotation curves for LSB galaxies.

The blue curve is the observed data and red is the curve obtained from theory. The average baryonic density of each galaxy is kept constant by keeping the value of $r_{c}$ in Eqn.(33) constant.

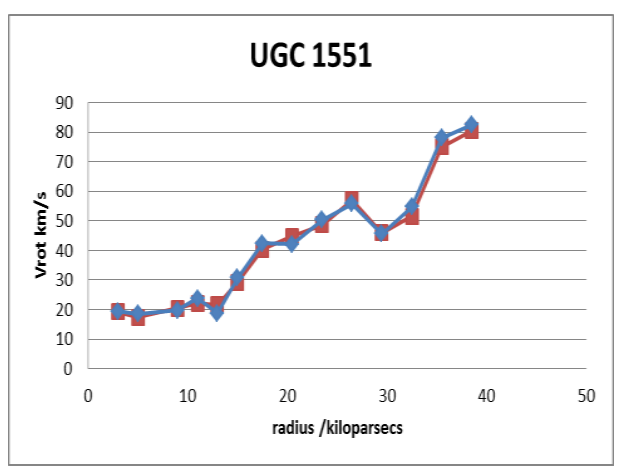

Fig.2

LSB galaxy UGC 1551. The blue curve is the observed data and red is the curve obtained from theory by adjusting the baryonic matter density appropriately along the radius by changing the value of $r_{c}$ in Eqn.(33). If the same procedure is applied to the above galaxies perfect fits are also obtained. 


\subsection{Preliminary test 2: The evolving Baryonic Tully -Fisher relation}

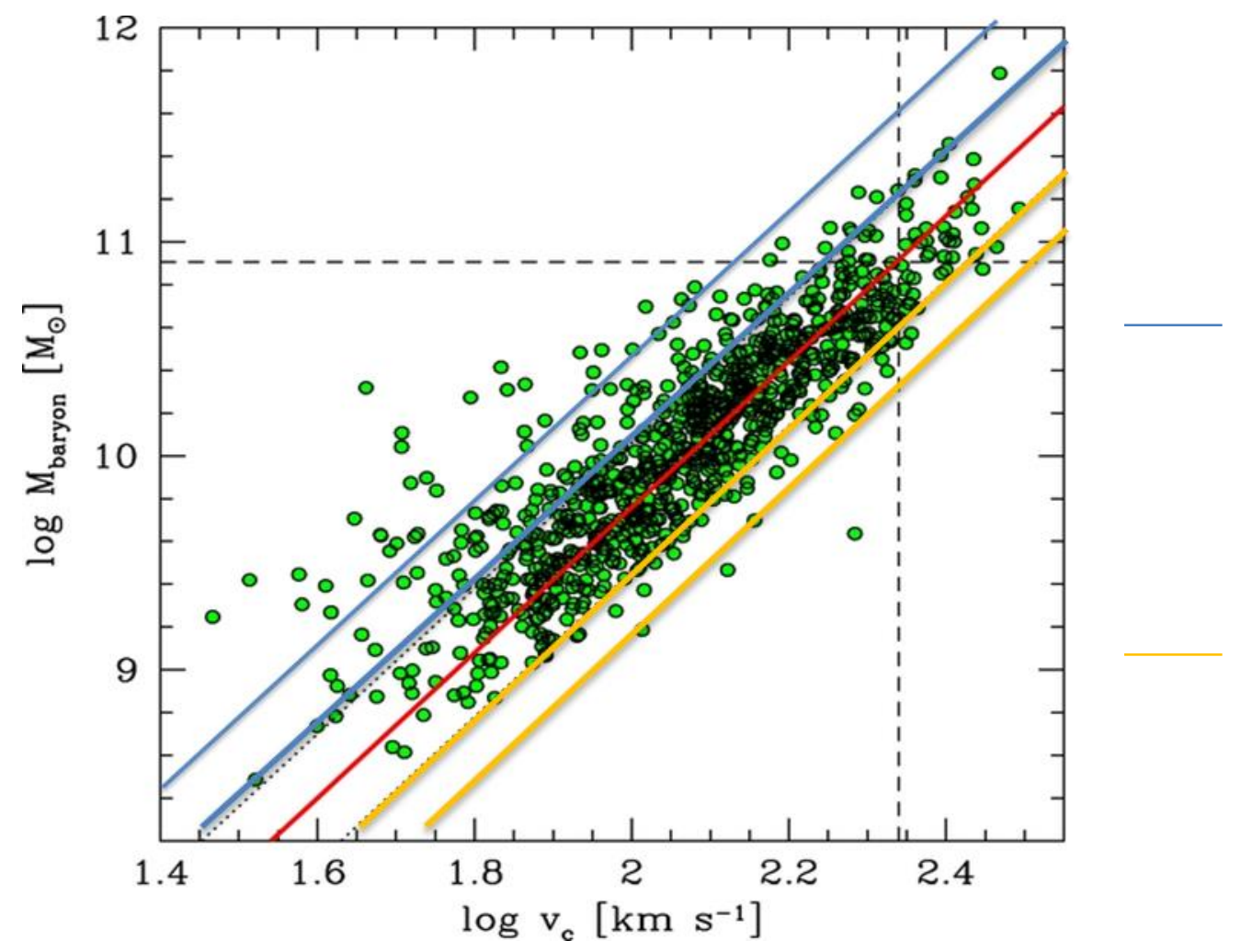

Young galaxies relative to red line galaxies

old galaxies relative to red line galaxies

Fig 3

- Horizontal lines: galaxies of same baryonic mass, different ages and different velocities.

- Vertical lines: galaxies of same velocity, different baryonic mass and different age.

- Diagonal lines: galaxies of same age, different baryonic mass and different velocities.

- Three line intersection: Galaxies of identical velocities, baryonic mass and age.

Primary image and data courtesy Dennis Zaritsky et al. 2014 AJ 147134 doi:10.1088/0004-6256/147/6/134

One salient feature of Eqn. (18) is an age dependent velocity relation for galaxies and galaxy clusters.

In Fig. 3 the relation is depicted from the following equation

$$
-\log M_{b}=-4 \log V_{c}+\log \left(G H_{0} c\right)+4 H_{0}\left(t-t_{e}\right) \log e
$$

Here $t_{e}$ is the age of the red line galaxies measured from the time they acquired dynamic equilibrium.

The above relation explains why galaxies of the same baryonic mass may have different rotational speeds and why satellite galaxy clusters have high orbital speeds despite the low baryonic mass content of the main cluster. 


\section{Gravitational Lensing Results}

Having passed the preliminary tests, we apply the Nexus Paradigm using Eqn.(23) and Eqn.(25) to determine the value of $n$, and $v_{n}$ from which we compute $a_{n}$ and $r_{n}$ from Eqn.(17) and Eqn.(19) for a spherically symmetric lensing system. The data is obtained from a sample of fifteen ERs lenses published on the CASTLES website (www.cfa.havard.edu/castles/). The calculations assume a flat universe in which $\mathrm{H}_{0}=69.6 \mathrm{~km} / \mathrm{s} / \mathrm{Mpc} \Omega_{\Lambda}=0.714 \Omega_{\mathrm{M}}=0.286$. The results are displayed in Table 1 . The terms $n, r_{n}, v_{n}$ and $a_{n}$ describe the quantized and dynamical states of space-time within the lensing system.

Table 1

\begin{tabular}{|c|c|c|c|c|c|c|}
\hline Lens & $\mathbf{D}_{\mathrm{ls}} / \mathrm{D}_{\mathrm{s}}$ & $\boldsymbol{\theta}_{E} / \operatorname{arcs}$ & $\mathbf{n}$ & $\mathbf{r}_{\mathbf{n}} / \mathbf{M p c}$ & $\mathrm{v}_{\mathrm{n}} / \mathrm{kms}^{-1}$ & $a_{n} / 10^{-10} m / s^{-2}$ \\
\hline $\begin{array}{l}\text { JVA } \\
\text { B1938+666 }\end{array}$ & 0.50 & 0.44 & 1948 & 2.27 & 154.0 & 0.0034 \\
\hline B0218+357 & 0.17 & 0.16 & 5508 & 0.80 & 54.5 & 0.0012 \\
\hline PG1115+080 & 0.64 & 1.16 & 1054 & 4.19 & 284.6 & 0.0063 \\
\hline B1608+656 & 0.35 & 1.14 & 1268 & 3.49 & 236.6 & 0.0052 \\
\hline $\begin{array}{l}\text { RXJ1131- } \\
1231\end{array}$ & 0.45 & 1.90 & 982 & 4.50 & 305.5 & 0.0067 \\
\hline Q0047-2808 & 0.58 & 1.35 & 1027 & 4.30 & 292.1 & 0.0064 \\
\hline $\begin{array}{l}\text { PMNJ0134- } \\
0931\end{array}$ & 0.37 & 0.37 & 2456 & 1.80 & 122.1 & 0.0027 \\
\hline $\begin{array}{l}\text { HE0230- } \\
2130\end{array}$ & 0.49 & 1.03 & 1231 & 3.62 & 243.7 & 0.0054 \\
\hline $\begin{array}{l}\text { CFR503.107 } \\
7\end{array}$ & 0.34 & 1.05 & 1581 & 2.80 & 189.8 & 0.0042 \\
\hline $\begin{array}{l}\text { HST15433+ } \\
5352\end{array}$ & 0.52 & 0.59 & 1640 & 2.69 & 182.9 & 0.0040 \\
\hline $\begin{array}{l}\text { MG1549+30 } \\
47\end{array}$ & 0.83 & 0.85 & 1081 & 4.09 & 277.5 & 0.0061 \\
\hline $\begin{array}{l}\text { PKS1830- } \\
211 \\
\end{array}$ & 0.34 & 0.50 & 2214 & 2.00 & 135.5 & 0.0030 \\
\hline $\begin{array}{l}\text { MG2016+11 } \\
2\end{array}$ & 0.33 & 1.76 & 1192 & 3.71 & 251.7 & 0.0055 \\
\hline Q2237+030 & 0.94 & 0.89 & 993 & 4.45 & 302.1 & 0.0066 \\
\hline $\begin{array}{l}\text { HE0435- } \\
1223\end{array}$ & 0.51 & 1.21 & 1156 & 3.82 & 259.5 & 0.0057 \\
\hline
\end{tabular}




\section{Discussion}

The results indicate that the orbital velocity of the constituents of a lensing system can be attributed to a globule of quantized vacuum energy called the Nexus graviton of density $\rho=n^{2} \rho_{D E}$ with a radius of dimensions in the order of a few mega parsecs. When in close proximity to other gravitons, tidal forces arise which transform the Nexus graviton from a spherical shape to an ellipsoid. The profile of the ellipsoid is an ellipse and the graviton radius becomes

$$
r_{n}=\frac{R_{n}}{1-e \cos \varphi}
$$

Where $e$ is the eccentricity.

Hence the orbital velocity becomes

$$
v_{n}=H_{0} r_{n}=\frac{H_{0} R_{n}}{1-e \cos \varphi}
$$

Where $R_{n}$ is the maximum radius of a spherically symmetric Nexus Graviton in the $n$-th quantum state. From Eqn.(20) we compute the vacuum energy density as

$$
\begin{aligned}
\rho=\frac{c^{2}}{v_{n}^{2}} \rho_{D E} & =\frac{R_{H}^{2}(1-e \cos \varphi)^{2} \rho_{D E}}{R_{n}^{2}} \\
& =n^{2}(1-e \cos \varphi)^{2} \rho_{D E}
\end{aligned}
$$

where $R_{H}$ is the Hubble radius. The deflection of light under these conditions becomes

$$
\alpha=(\theta-\beta) D_{l s} / D_{s}=4 / n^{2}(1-e \cos \varphi)^{2}
$$

Here we notice an increase in vacuum energy density with a decrease in curvature radius. The density profile is thus stratified in quantized concentric radii $r_{n}=R_{H} / n$ with a maximum radius at $\mathrm{R}_{\mathrm{N}}<\mathrm{R}_{\mathrm{H}}$ and the minimum at $\mathrm{R}_{\min }=\mathrm{R}_{\mathrm{H}} / 10^{60}$. Space-time in the inner core of the Nexus graviton is therefore flat as described by Eqn.(11). In the LCDM paradigm, the gravitational lensing at galactic and cosmic scales is an effect arising largely due to the presence of the hypothetical DM in the lensing system and so it can be used to constrain the DM mass model of lenses as 
in Fig.4. In the Nexus Paradigm, the gravitational lensing can be used to constrain the value of the quantum state $n$ of space-time within the lensing system also as in Fig.4. The relationship between the quantum state of space-time and the hypothetical DM is expressed in Eqn.(40) if $M(r)$ is considered as the DM distribution.

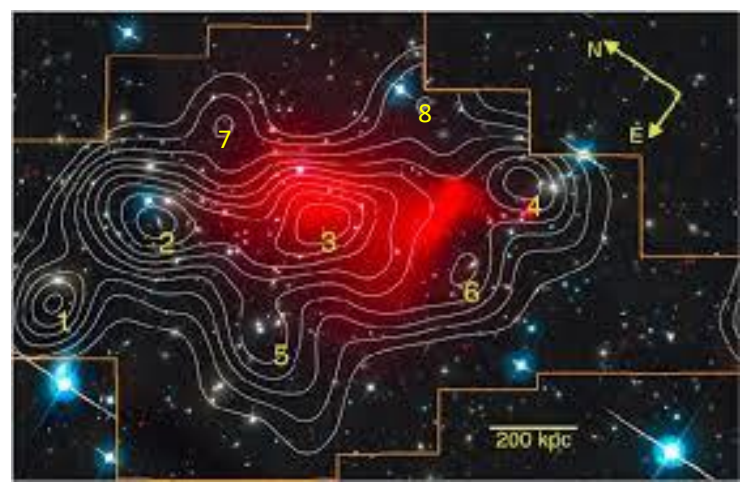

Fig.4 The Abell 520 Cluster

The Abell 520 cluster in Fig.4 shows eight interacting Nexus gravitons. The interaction deforms each graviton into an ellipsoid of cross section described by Eqn. (37).

By comparing the quantized metric of Eqn.(11) with Schwarzschild metric we notice that the quantum state of space-time around baryonic matter increases with distance from the mass

$$
\frac{2}{n^{2}}=\frac{2 G M(r)}{c^{2} r}
$$

Such that

$$
n^{2}=\frac{c^{2} r}{G M(r)}
$$

Thus the curvature of space-time in the inner core of the Nexus graviton where high quantum states are prevalent is flat as described by Eqn.(11) and is only curved by the presence of baryonic matter and not by the increased vacuum energy density. This solves the so called corecusp problem of astrophysics.

If the Nexus graviton is surrounded by multiple gravitons tugging on it gravitationally, then the eccentricity becomes a function of the azimuthal angle $\psi$. Two gravitons of radii $\mathrm{r}_{\mathrm{n}}=\mathrm{f}(\psi)$ and $\mathrm{r}_{\mathrm{s}}=\mathrm{g}(\psi)$ will intersect when $\mathrm{f}(\psi)=\mathrm{g}(\psi)$. 


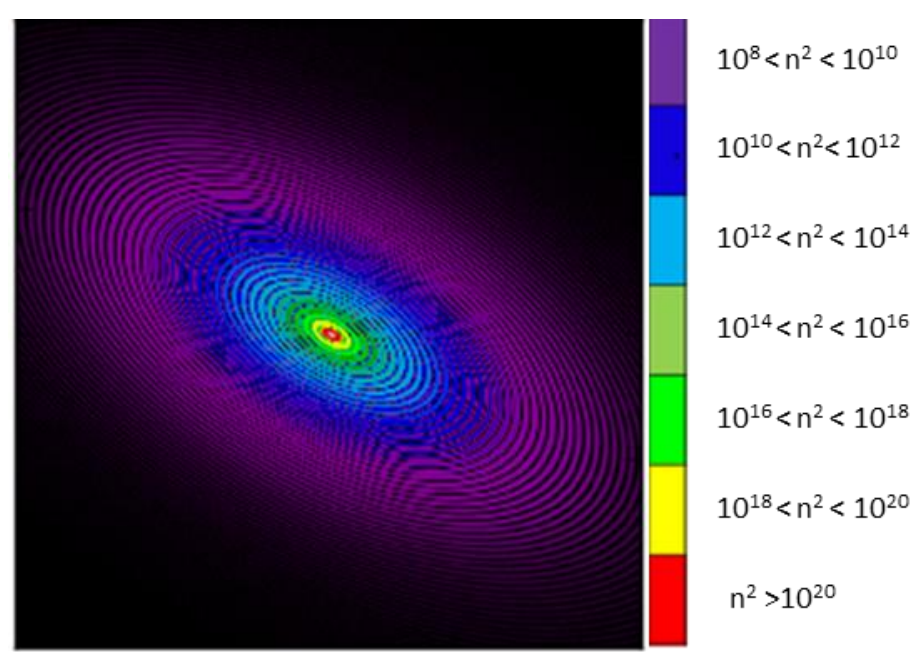

Fig.5

The vacuum energy density profile $\rho=n^{2}(1-e \cos \varphi)^{2} \rho_{D E}$ of an ellipsoid Nexus graviton in units of $\rho_{D E}$. The lensing potential increases with radial distance in accordance with Eqn.(38).

A prolate spheriod Nexus graviton depicted in Fig. 5 can be traced out by far out satellite galaxies.

Their radial velocities should behave according to the rule $v_{n}=\frac{H_{0} R_{n}}{1-e \cos \varphi}$ relative to the central galaxy. The Milky Way satellites can be used to probe this behaviour.

\section{Conclusion}

Quantum gravity can explain phenomena attributed to DM and DE. Gravity in the Nexus Paradigm is considered as a region of low vacuum energy density. Thus a zone with DM is explained as a region of low vacuum energy density or low space-time quantum state. If we attribute galaxy rotation curves and cluster dynamics to the Nexus graviton instead of exotic DM which despite extensive searches has still not yet materialized, we can simplify our calculations in predicting the behaviour of such systems. Currently, the preferred DM paradigm requires a variety of density profiles to explain such dynamics. Moreover each profile has its difficulties which demand the DM model to be fine-tuned to fit different scenarios. 


\section{Acknowledgments}

We gratefully appreciate the funding and support from the Department of Physics and Astronomy at the Botswana International University of Science and Technology (BIUST).

\section{References}

- Archambault S et al. 2009 [PICASS0 Collaboration] Phys. Lett. B 682(2) 185-192.

- Akerib D S et al ( LUX Collaboration) 2017 Phys.Rev.Lett. 118021303

- Boller T H 1989 Astron. Nachr 310,362

- Bullock J S \& Boylan-Kolchin 2017 Annual Review of Astronomy and Astrophysics 55343 387

- Capozziello S 2002 Int. J. Mod. Phys. D $11483-491$

- Clowe D et al 2006 Astrophys.J. 648(2) (2006) 109-113.

- Clowe D et al, 2012 Astrophys.J. 758,128

- Cushman P et al 2013, Preprint hep-ex.1310.8327v2

- De Blok WJG 2009 preprint [astro-ph.CO] 0910.3538v1

- $\quad$ Ellis R S 2010 Phil. Trans. R. Soc. A $2010368967-987$

- Federico L, McGaugh S and Schombert J M 2015 Preprint[astrp-ph.GA] 1512.04543v1

- Ferrero I, Abadi M G, Navarro F J, Sales and V J, Gurovich S 2012 MNRAS, 425 2817-2823

- Goodman M and Witten E 1985 Phys.Rev. D 31(2) 3059.

- Israel M et.al, $2014 A \& A \mathbf{5 6 4 , 1 2 9}$

- Jauzac M et al 2015 MNRAS 446,4132-4147

- Jee M J, Mahdavi A, Hoekstra H, Babul A, Dalcanton J J, Carroll P, and Capak P 2012 The Astrophysical Journal 747 (96)

- Jee M J, Mahdavi A, Hoekstra H and Babul A 2014 The Astrophysical Journal 783 (78)

- Kitching T et al, 2015 Astronomy and Computing 10 9-21

- Kuzio de Naray et al. 2006, ApJS, 165, 461

- Kuzio de Naray et al. 2008, ApJ, 676, 920

- Leauthaud A et al 2015 MNRAS 446 1874-1888

- Lee J, and Komatsu E 2010 Astrophysical Journal 718 60-65

- Li B, Sotiriou T P and Barrow J D 2011 Phys. Rev. D 83104017.

- Marongwe S 2015 Int. J. Geom. Methods Mod. Phys. 12(4) 1550042,

- Marongwe S 2017 Int. J. Mod. Phys. D 261750020

- Massey R et al, 2013 MNRAS 429, 661-678

- McGaugh S S 2005 The Astrophysical Journal 632 (2)

- McGaugh S 2011Preprint [astro-ph] 1107.2934v2

- McGaugh 2014 S Canadian Journal of Astrophysics 93(2) 250-259

- Milgrom M 1983 Astrophys. J. 270 371-389.

- Navarro J , Carlos F S and Eke V R 1996 MNRAS. 283(3) L72-L78

- Olmo G J 2005 Phys. Rev. D 72083505

- Sangalard V et al. 2005 Phy. Rev. D 71(12) 122002.

- Se-Heon O et al 2015 The Astronomical Journal 149 (6)

- Tan A et al (Panda X-II Collaboration) 2016 Phys.Rev.Lett. 117121303 
- $\quad$ The CDMS II Collaboration 2010 Science 327(5973) 1619-1621.

- Thompson R, Dave R and Nagamine K 2014 Preprint [astro-ph.CO]1410.7438v2

- Van den Bosch F C and Swaters R A 2001 MNRAS, 325 (3) 1017-1038

- Zhandov V I, Alexandrov A N, Fedrova EVK and Sliusar V M 2012 Astronomy and Astrophysics 2012, Article ID 906951

- Zwicky F 1937 ApJ86,217 